\title{
QUASI-1500-YEAR-CYCLE SIGNAL IN LENGTH-OF-DAY CHANGE
}

\author{
Lihua MA \\ National Astronomical Observatories, Chinese Academy of Sciences, \\ Beijing, P.R. China \\ e-mail:mlh@nao.cas.cn
}

\begin{abstract}
Length-of-day (LOD) change, i.e., variations in Earth's rotation rate, includes the long-term slowdown trend, as well as periodic and irregular fluctuations. The current continuous sequence of the LOD change covers a time span of $<400$ years. Using astronomical records in ancient historical documents, combined with a modern astronomical ephemeris, it is possible to obtain ancient LOD change. Some scholars have given a discontinuous LOD data series for the past 4000 years. In this paper, the author uses the Lomb-Scargle periodogram to study the LOD series and finds a significant quasi-1500-yearcycle signal. Furthermore, with weighted wavelet Z-transform, time-varying characteristics of the cycle in the LOD change are obtained.
\end{abstract}

Keywords: length-of-day change, Lomb-Scargle periodogram, weighted wavelet Z-transform

\section{INTRODUCTION}

Variations of Earth's rotation characterize the geodynamical processes of various layers of the Earth system. Earth's rotation is closely related to local movements of the Earth (including movement of the solid Earth, as well as flows of sea water and atmosphere). The variations are composed of changes in the direction of the pointing axis and variations of the Earth's rotation rate characterized by the length-of-day (LOD) change. In recent years, advanced astronomical observation facilities have greatly improved the accuracy of astrogeodynamical observations and enabled people to have more refined understanding of some geodynamical processes. The LOD change includes the long-term slowdown trend, periodic fluctuations, and irregular fluctuations. The fluctuations in the LOD change over several days and on interseasonal and interannual time scales mainly originate from variations of atmospheric axial angular momentum (Gross et al., 2004; Ma and Han, 2006; Ma et al., 2006). Recent studies have shown that excitation of decadal fluctuations in the LOD change is mainly due to solar activity and variations in the mean sea level (Chapanov and Gambis, 2010; Chapanov et al., 2008, 2010, 2015; Ron et al., 2012).

Since the middle of the 20th century, some proxies that indicate geodynamical processes covering thousands, tens of thousands, and even millions of years have provided people opportunities to understand the Earth system. There are a large number of historical documents that make available astronomical observations from ancient times. Based on this, the ancient LOD change can be deduced. At present, combined with ancient and modern astronomical observations, the continuous LOD series has a time span of $<400$ years. To do an in-depth study of long-period fluctuations in the LOD change, a longer sequence of the 
LOD change is needed. Stephenson et al. (2016) integrated more ancient astronomical records to obtain an LOD data series for the past 4000 years. However, the LOD series has uneven time intervals. Here, the Lomb-Scargle periodogram and weighted wavelet Z-transform (WWZ) methods are used to study the LOD sequence, with ephemeris on the long-period fluctuations in the LOD change.

\section{DATA AND METHOD}

\subsection{Data de scription}

Astronomical records in historical documents describe local observations in ancient time. With a modern astronomical ephemeris, ancient astronomical observations recorded in historical documents can be theoretically calculated. Studies have shown that there is a difference of observable conditions between theoretical calculations and historical records. The earlier the ancient historical records, the more remarkable the difference is. The fundamental reason is the variation in Earth's rotation. Combined with the difference, one can easily calculate the LOD change in ancient times. Stephenson et al. (2016) synthesized some ancient astronomical observations and obtained the discontinuous LOD series from $2001 \mathrm{BCE}$ to $2016 \mathrm{CE}$. The sampling intervals before $1600 \mathrm{CE}$, from $1600 \mathrm{CE}$ to $1800 \mathrm{CE}$, from 1800 $\mathrm{CE}$ to $1950 \mathrm{CE}$, and after $1950 \mathrm{CE}$ are 100 years, 10 years, 1 year, and 0.5 years, respectively. The LOD series is shown using a black solid line in Figure 1. There is a significant long-term slowdown trend in the LOD change. The linear trend with $+1.78 \mathrm{~ms} \mathrm{cy}^{-1}$ (milliseconds per century) is given with a red dashed line in Figure 1. Additionally, there are long-period fluctuations in the LOD series. To facilitate analysis of the long-term fluctuations, the trend of long-term slowdown is removed from the LOD series. The new sequence is plotted with blue solid line in Figure 1. This LOD series without linear trend is analyzed in the following sections.

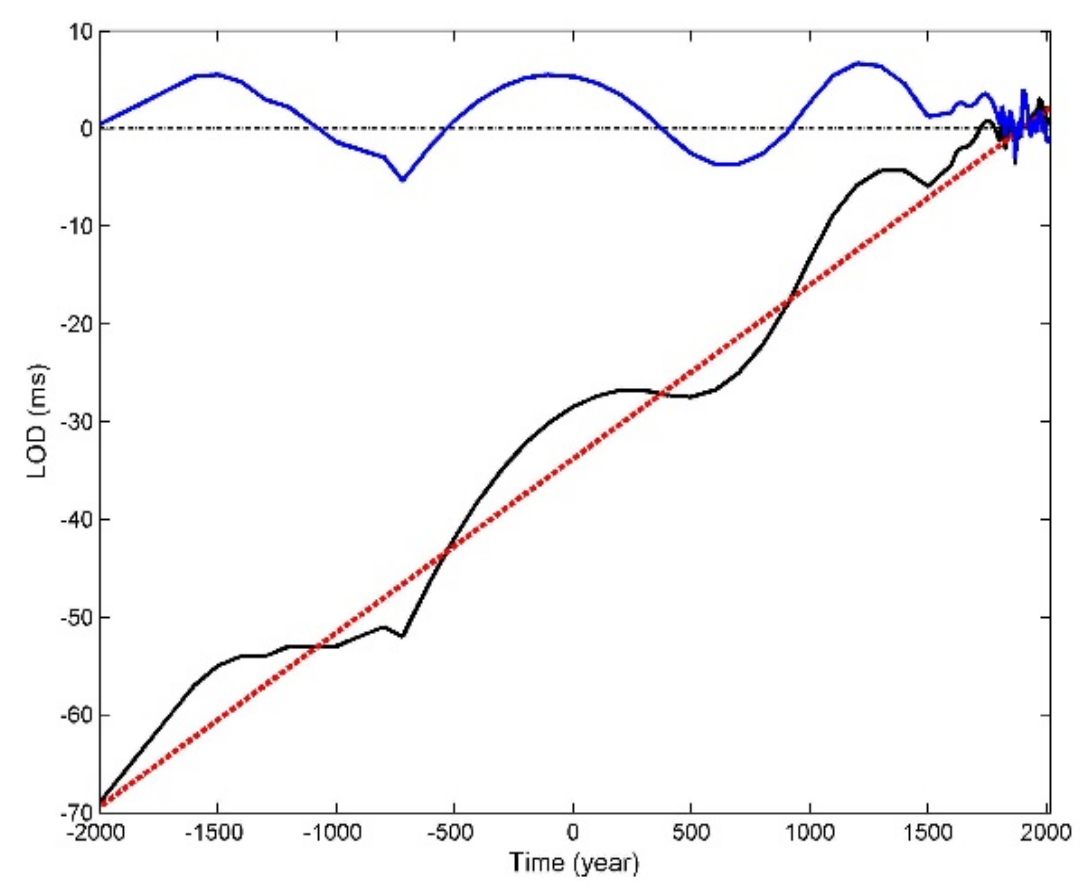

Figure 1. The LOD series from $2001 \mathrm{BCE}$ to $2016 \mathrm{CE}$ 


\subsection{Lomb-Scargle pe riodogram}

Considering that actual observations are affected by weather, observation equipment, and other conditions, observations are discontinuous and uneven in the time domain. Conventional periodic analysis techniques require that data series is uniformly sampled; so, uneven observations need to be interpolated or zero-filled. However, interpolation or zero-filled data can produce false information. Least-squares (Laguna et al., 1998) and phase dispersion minimization (Stellingwerf, 1978) can deal with uneven data. Here, the Lomb-Scargle periodogram approach is adopted. The periodogram can find periodic signals from uneven observation series (Lomb, 1976; Scargle, 1982). Based on the principle of discrete Fourier transform, the Lomb-Scargle periodogram decomposes some data series into a series of linear combinations of sine (cosine) functions and transforms the signal characteristics from the time domain to the frequency domain.

Consider an observation sequence $X\left(t_{j}\right), j=1,2, \ldots, N$. The Lomb-Scargle periodogram estimates the power spectrum $P(\omega)$ of the signal by calculating the function with the frequency $\omega$.

$$
P(\omega)=\frac{1}{2}\left\{\frac{\sum_{j=1}^{N}\left(X\left(t_{j}-\mu\right) \cos \omega\left(t_{j}-\tau\right)\right)^{2}}{\sum_{j=1}^{N} \cos ^{2} \omega\left(t_{j}-\tau\right)}+\frac{\sum_{j=1}^{N}\left(X\left(t_{j}-\mu\right) \sin \omega\left(t_{j}-\tau\right)\right)^{2}}{\sum_{j=1}^{N} \sin ^{2} \omega\left(t_{j}-\tau\right)}\right\},
$$

where $\mu$ represents the mean value of the sequence $X\left(t_{j}\right)$, and $\tau$ is calculated using the following formula:

$$
\tan (2 \omega \tau)=\frac{\sum_{j=1}^{N} \sin 2 \omega t_{j}}{\sum_{j=1}^{N} \cos 2 \omega t_{j}}
$$

In order to investigate the quality of $P(\omega)$, the significance level $\alpha$, representing the probability of the spectrum, is used for evaluation. If $\alpha$ is equal to 0.1 , the trust extent of the spectrum is $90 \%$.

$$
\alpha=1-\left(1-e^{-\eta}\right)^{k}
$$

where $\eta$ is the estimated spectrum value, and $k$ represents the resolution of the spectrum. The number of sampling points in the frequency domain depends on the resolution required during the calculation.

\subsection{WWZ method}

Wavelet transform appeared in recent years, and it gradually refines the signal in multiple scales through expansion and translation operations, which can automatically adapt to requirements of time-frequency signal analysis and can focus on details of the signal (Daubechies, 1992; Foufoula-Georgiou and Kumar, 1994). Using the wavelet transform, it is possible to approximate discrete signals with local characteristics and more comprehensively reflect changes of the original signal on a certain time scale. Foster (1996a, 1996b) proposed a WWZ algorithm, which can directly detect the period of uneven sampling time series. This method reveals the change of series on a certain time scale through localized analysis of time (space) frequency. Based on the principle of wavelet transform, the WWZ algorithm can directly detect periods of uneven time series. The WWZ transform is defined as follows: 


$$
Z=\frac{\left(N_{e f f n}-1\right) V_{y}}{2 V_{x}},
$$

where $N_{\text {effn }}$ is the number of effective points; $V_{x}$ is the weighted variation of the data, satisfying the condition

$$
V_{x}=(x, x)-(1, x)=\frac{\sum_{i} \omega_{i}\left(x\left(t_{i}\right)\right)^{2}}{\sum_{j} \omega_{j}}-\left[\frac{\sum_{i} \omega_{i}\left(x\left(t_{i}\right)\right)}{\sum_{j} \omega_{j}}\right]^{2} .
$$

$V_{y}$ is the weighted variation of mode functions, satisfying the following:

$$
V_{y}=(y, y)-(1, y)=\frac{\sum_{i} \omega_{i}\left(y\left(t_{i}\right)\right)^{2}}{\sum_{j} \omega_{j}}-\left[\frac{\sum_{i} \omega_{i}\left(y\left(t_{i}\right)\right)}{\sum_{j} \omega_{j}}\right]^{2} .
$$

Considering the fixed parameters $\omega$ and $\tau$, the WWZ transform obeys the chi-square distributions, and the degrees of freedom are $N_{\text {effi }}-3$ and 2, respectively.

\section{LOMB-SCARGLE PERIODOGRAM}

The Lomb-Scargle periodogram of the LOD series is calculated, and the results are plotted in Figure 2. It can be seen that there is a significant quasi-1500-year cycle in the LOD time series. The significance level of the periodic signal is $>99.9 \%$, corresponding to $\alpha=0.001$.

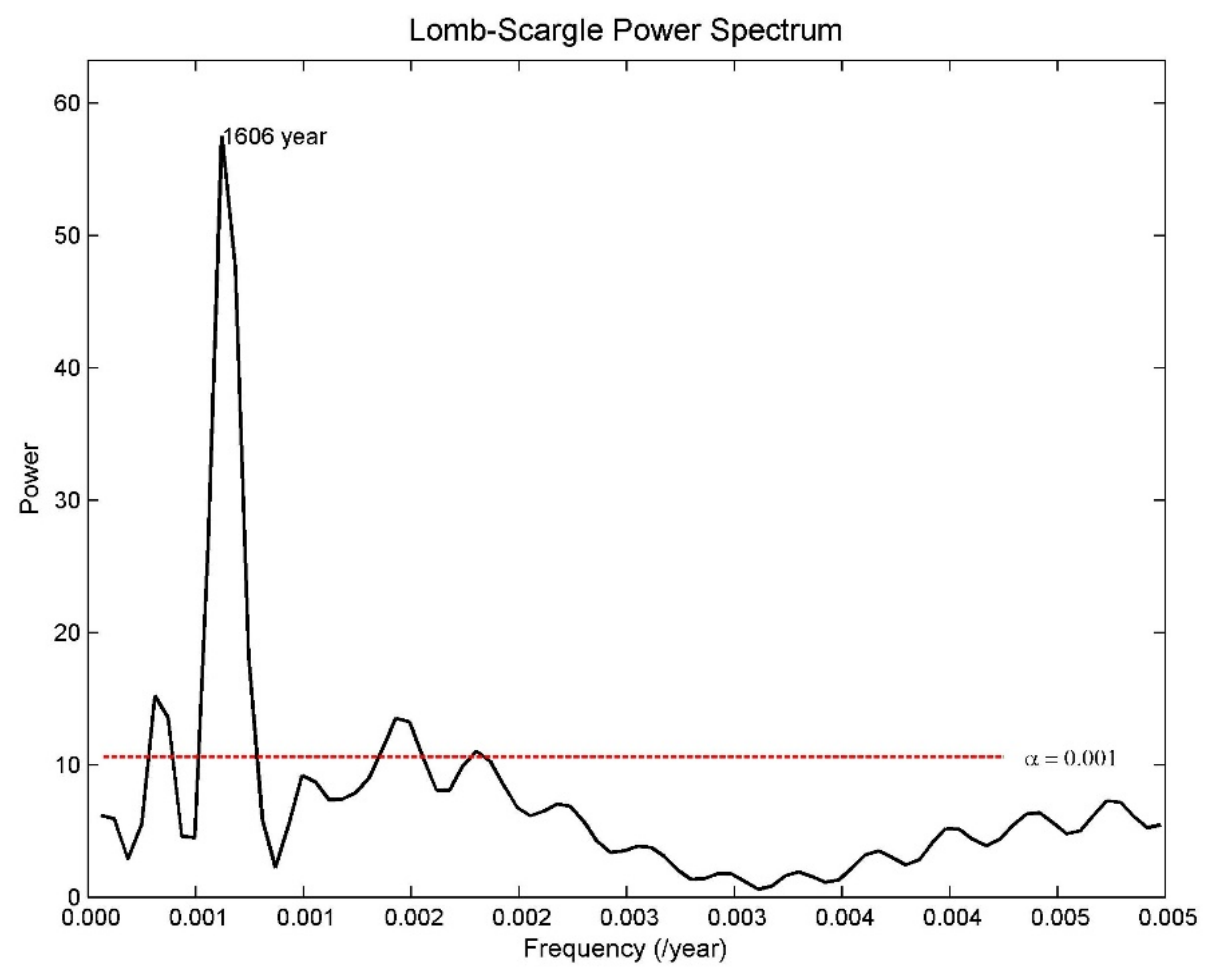

Figure 2. The Lomb-Scargle periodogram of the LOD series 


\section{THE WWZ ANALYSIS}

The above Lomb-Scargle periodogram only shows the average effects of periodic fluctuations in the whole time span of the LOD time series. Here the WWZ transform is used to analyze the series and the corresponding power spectrum is plotted in Figure 3. It can be seen that the WWZ analysis gives the detailed spectrum structure of some periodic signals. There are significant quasi-1500 years cycle signals in the LOD series. Furthermore, significant timevarying characteristics of the cycle can be found. From the beginning of the LOD series, there are signs of a quasi-1500 years cycle. This cycle is obvious between $2000 \mathrm{BCE}$ and $1000 \mathrm{CE}$, and the most prominent cycle signals appear between $1000 \mathrm{BCE}$ and $300 \mathrm{BCE}$.

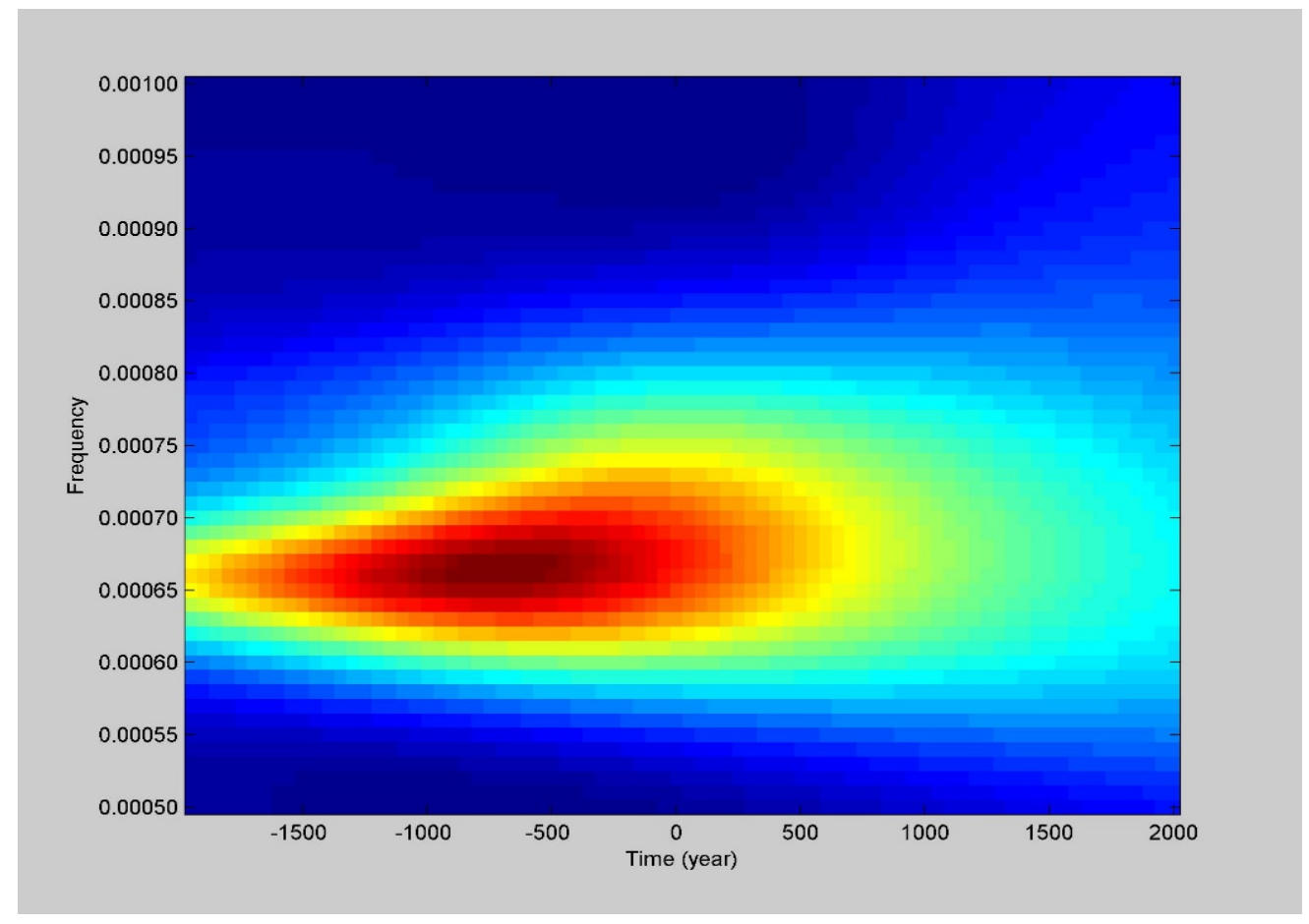

Figure 3. The WWZ power spectrum of the LOD series

Here, we calculate the sum of the WWZ power for each calculated value of the frequency; the normalized global WWZ power distribution in the frequency domain can be obtained (Figure 4). The figure also shows the quasi-1500-year-cycle signals in the LOD change. 


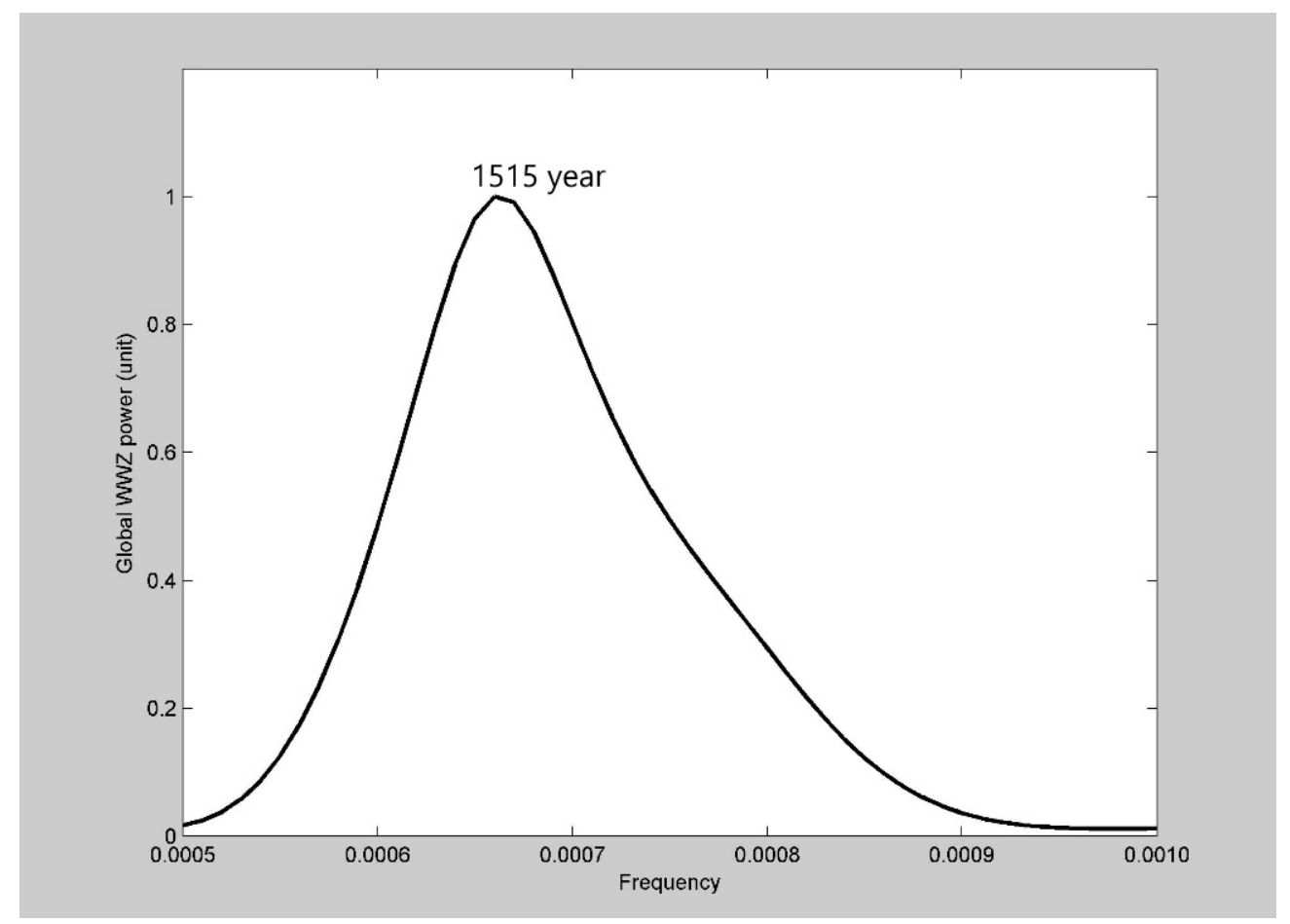

Figure 4. The global WWZ power spectrum

To investigate the changes in the quasi-1500-year cycle with time, we select a time scale from 1200 to 1800 years as the range of the cycle length for the quasi-1500-year cycle and then calculate the sum of the WWZ power in the selected time scale at a certain moment. In this way, we obtain the fluctuations of the quasi-1500-year cycle over the whole time span. The results with normalized WWZ power are plotted in Figure 5. The figure further shows the time-varying characteristics of the quasi-1500-year cycle, consistent with the WWZ power spectrum.

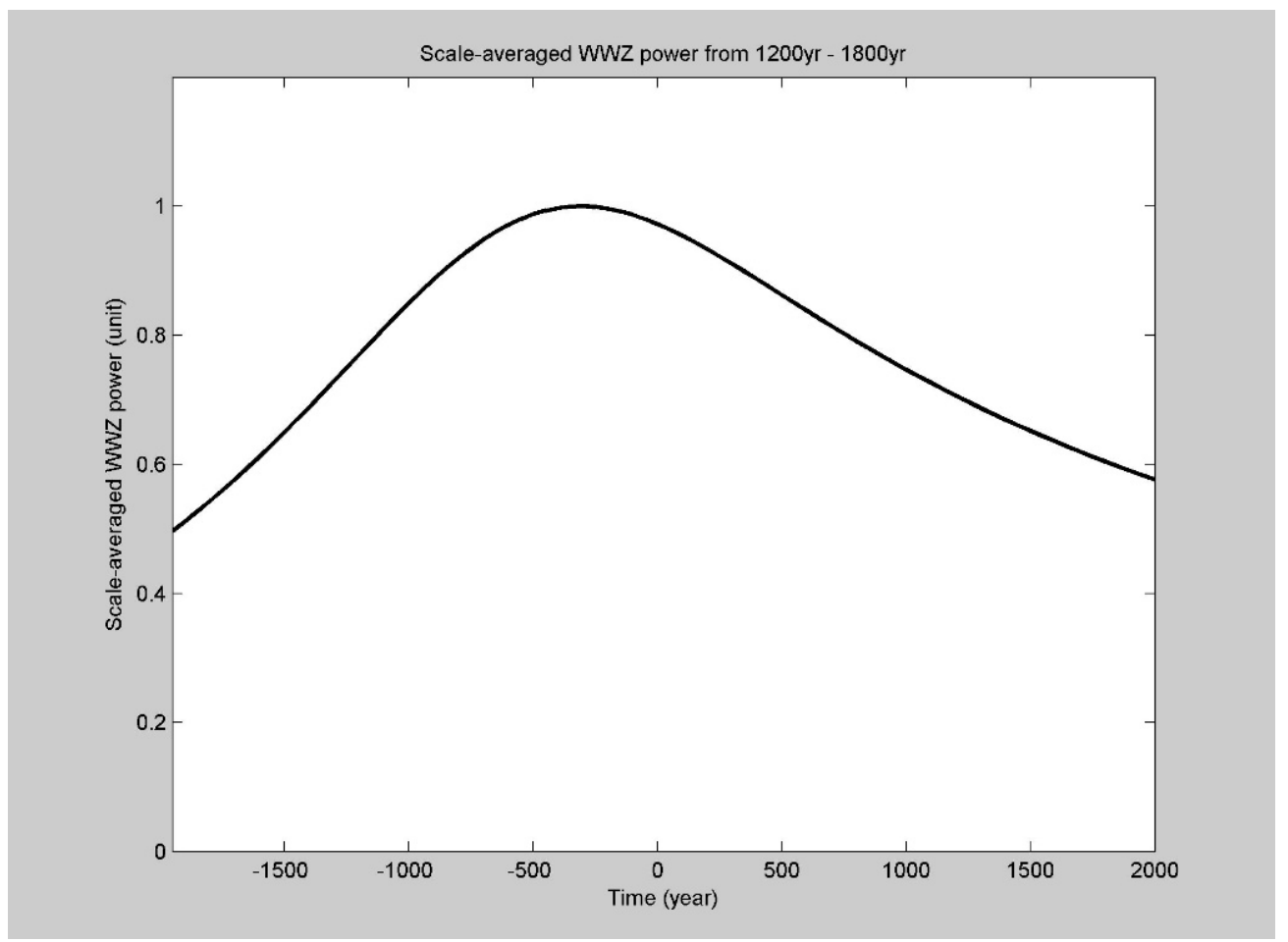

Figure 5. The WWZ power spectrum of the LOD series from 1200- to 1800-year time scales 


\section{CONCLUSIONS}

The LOD series with uneven time intervals from 2001 BCE to 2016 CE from historical documents is analyzed in this work, with ephemeris on long-period fluctuations. The LombScargle periodogram and WWZ transform show that there is a significant quasi-1500-year cycle in the LOD series. Meanwhile, the cycle signal shows obvious time-varying characteristics. The cycle is the strongest from $1000 \mathrm{BCE}$ to $300 \mathrm{BCE}$. The physical mechanism that produces this cycle needs to be studied in the future. Additionally, considering that the discontinuous LOD series analyzed in this work are taken from ancient historical documents, the data source is relatively single, and there are certain errors in the records. It will be more meaningful if this could be combined with long time-span LOD series obtained from other related geodynamical processes. Additionally, Bond et al. (2001) and Debret et al. (2007) have identified a quasi-1500-year solar cycle that has had a significant influence on North Atlantic climate throughout the Holocene. The cycle is also known as the Dansgaard-Oeschger 1470-year cycle (Schulz, 2002). The quasi-1500-year cycle existing in these various geophysical series may be related to the rotation of the Earth. The detailed analysis of the physical mechanism will be included in an extended article in the future.

Acknowledgements. The author is grateful to M. Emre Aydin for providing a weighted wavelet Z-transformation analysis program. The author also thanks the anonymous reviewers for helpful comments, which improved the manuscript greatly. This work has made use of the Astrophysics Data System (National Aeronautics and Space Administration, USA).

\section{REFERENCES}

Bond G., Kromer B., Beer J., Muscheler R., Evans M. N., Showers W., Hoffmann S., LottiBond R., Hajdas I., Bonani G. (2001) Persistent solar influence on North Atlantic climate during the Holocene, Science, Vol. 294, No. 5549, 2130-2136.

Chapanov Ya., Gambis D. (2010) Solar-terrestrial energy transfer during sunspot cycles and mechanism of Earth rotation excitation, Proceedings of the IAU, Volume 5, Symposium S264, Solar and Stellar Variability: Impact on Earth and Planets, 404-406.

Chapanov Ya., Ron C., Vondrák J. (2015) Millennial cycles of mean sea level excited by Earth's orbital variations, Acta Geodynamica et Geomaterialia, Vol. 12, No. 3, 259-266.

Chapanov Ya., Vondrák J., Ron C. (2008) Decadal oscillations of the Earth rotation, AIP Conference Proceedings, Vol. 1043, 197-200.

Chapanov Ya., Vondrák J., Ron C. (2010) Common 22-year cycles of Earth rotation and solar activity, Proceedings of the IAU, Volume 5, Symposium S264, 407-409.

Daubechies I. (1992) Ten Lectures on Wavelets, SIAM, Philadelphia.

Debret M., Bout-Roumazeilles V., Grousset F., Desmet M., McManus J.F., Massei N., Sebag D., Petit J.-R., Copard Y., Trentesaux A. (2007) The origin of the 1500-year climate cycles in Holocene North-Atlantic records, Climate of the Past, Vol. 3, No. 4, 569-575.

Foster G. (1996a) Time series analysis by projection. I. statistical properties of Fourier analysis, The Astronomical Journal, Vol. 111, No. 1, 541-554.

Foster G. (1996b) Wavelets for period analysis of unevenly sampled time series, The Astronomical Journal, Vol. 112, No. 4, 1709-1729.

Foufoula-Georgiou E., Kumar P. (1994) Wavelets in Geophysics, Academic Press, San Diego. 
Gross R.S., Fukumori I., Menemenlis D., Gegout P. (2004) Atmospheric and oceanic excitation of length-of-day variations during 1980-2000, Journal of Geophysical Research, Vol. 109, B01406.

Laguna P., Moody G.B., Mark R.G. (1998) Power spectral density of unevenly sampled data by least-square analysis: Performance and application to heart rate signals, IEEE Transactions on Biomedical Engineering, Vol. 45, No. 6, 698-715.

Lomb N.R. (1976) Least-squares frequency analysis of unequally spaced data, Astrophysics and Space Science, Vol. 39, 447-462.

Ma L.H., Han Y.B. (2006) Atmospheric excitation of time variable length-of-day on seasonal scales, Chinese Journal of Astronomy and Astrophysics, Vol. 6, No. 1, 120-124.

Ma L.H., Liao D.C., Han Y.B. (2006) Atmospheric and oceanic excitations to LOD change on quasi-biennial time scales, Chinese Journal of Astronomy and Astrophysics, Vol. 6, No. 6, 759-768.

Ron C., Chapanov Ya., Vondrák J. (2012) Solar excitation of bicentennial Earth rotation oscillations, Acta Geodinamica et Geomaterialia, Vol. 9, No. 3, 259-268.

Scargle J.D. (1982) Studies in astronomical time series analysis. II - Statistical aspects of spectral analysis of unevenly spaced data, The Astrophysical Journal, Vol. 263, 835-853.

Schulz M. (2002) On the 1470-year pacing of Dansgaard-Oeschger warm events, Paleoceanography, Vol. 17, No. 2, 1014.

Stellingwerf R.F. (1978) Period determination using phase dispersion minimization, The Astrophysical Journal, Vol. 224, 953-960.

Stephenson F.R., Morrison L.V., Hohenkerk C.Y. (2016) Measurement of the Earth's rotation: $720 \mathrm{BC}$ to AD 2015, Royal Society's Proceedings A, 472, 20160404.

Received: 2021-02-28

Reviewed: 2021-03-15 (undisclosed name) and 2021-04-27 (Y. Chapanov)

Accepted: 2021-05-31 